\title{
La démarche diagnostique des troubles cognitifs chez l'âgé*
}

Marion Droz Mendelzweig ${ }^{a}$, Armin von Gunten ${ }^{d}$, Claire Newman ${ }^{b}$, Florence Galland Lainic, Krzysztof Skuza

a Prof. HES-SO, anthropologue, Dr sciences sociales et politiques; ${ }^{b}$ MSc psychologie clinique et psychologue FSP; ${ }^{\circ}$ Dr sciences sociales, ethnologue - Haute Ecole de la Santé La Source, Lausanne; ' Prof., spécialiste FMH en psychiatrie générale et gériatrique, Service Universitaire de Psychiatrie de l'Age Avancé (SUPAA), CHUV, Lausanne; e Prof. HESAV, soziologue et psychologue clinique, Dr sciences sociales, Haute Ecole de Santé Vaud (HESAV), Lausanne

\section{Summary}

Cet article retrace une recherche qualitative pluridisciplinaire. Les cinq auteurs - anthropologues, médecin psychiatre, psychologue et sociologue ont développé une collaboration autour d'une problématique récurrente chez les personnes âgées: les troubles cognitifs associés à l'âge, les difficultés à les diagnostiquer et à communiquer le diagnostic aux patients et à leurs proches.

Huit patients et leurs proches se sont rencontrés séparément lors de deux séries d'entretiens sur l'annonce du diagnostic Mild Cognitive Impairment $(\mathrm{MCl})$. Les résultats montrent que la communication du diagnostic $\mathrm{MCl}$ n'insuffle pas un tournant dans la trajectoire des patients. Les propos des experts semblent n'avoir qu'un faible impact sur la compréhension qu'ont les patients et leurs accompagnants de la santé mentale des sujets diagnostiqués.

\footnotetext{
* Troisième place du «Call for Paper in Medical Humanities» (cf. cadre à la page 178.)
}

\section{Introduction}

Entre 2011 et 2013, une étude pluridisciplinaire a été menée auprès de huit patients et de leurs proches ayant reçu un diagnostic de Mild Cognitive Impairment $(\mathrm{MCI})^{1}$ dans le cadre des "consultations mémoire» dans le canton de $\mathrm{Vaud}^{2}$. Son but: voir quels sont les possibles effets de la définition experte des troubles de mémoire sur les intéressés et sur les proches qui ont accompagné, voire initié la sollicitation de l'avis expert sur les troubles. L'orientation épistémique de cette étude considère la représentation qu'ont les individus de leur santé comme une production de sens qui s'accomplit notamment, mais pas exclusivement, au travers d'interactions sociales. Elle suppose la consultation du corps médical que partiellement influente sur ces interactions [2]. Les statuts et les rôles sociaux des experts influencent l'attention accordée aux discours relatifs à la définition de l'état de santé ainsi que la conceptualisation de ce qui est tenu pour vrai par les sujets diagnostiqués. Toutefois, l'idée que se font les patients et les proches d'un état de santé n'est pas uniquement fonction de ce qui leur a été communiqué lors de l'an- nonce du diagnostic. Les facteurs biomédicaux, psychiques et sociaux, tant conscients qu'inconscients, s'entremêlent intimement dans la construction de leur réalité.

Cette posture épistémique s'intéresse donc au diagnostic en tant qu'énoncé expert sous-tendant les représentations de la santé. Elle attribue à la communication médicale un rôle de tournant autour duquel patients et proches structurent leur perception, introduisant dans leurs récits de la maladie un ordonnancement entre un avant l'officialisation de la maladie, soutenue par l'annonce du diagnostic, et un après.

Dans un contexte de prégnance de la maladie d'Alzheimer (MA) dans les imaginaires du vieillissement $[3,4]$, le postulat à la base de cette étude était qu'un diagnostic MCI pouvait avoir un effet performatif sur les personnes diagnostiquées et sur leurs proches par l'influence qu'il pouvait exercer sur leur manière d'appréhender leurs perspectives de vie. L'association entre les troubles de mémoire à l'origine de leur venue à la consultation et la crainte d'une possible future MA devait, selon nous, peser sur les postures des patients et des proches face à la crainte de la maladie. Notre approche postule que les interprétations de sens commun données au déclin cognitif associé à l'avancée en âge s'élaborent dans le cadre de schémas de représentations ouverts à la créativité individuelle, mais néanmoins contraints par un nombre restreint de formes possibles de narrations [5]. La poly-influence sur les schémas de représentation que les sujets ont de leur santé, auxquels l'annonce du diagnostic participe, devait pouvoir se percevoir en mettant en évidence par le biais d'entretiens qualitatifs le processus d'élaboration conceptuelle, de catégorisation et de mise en récit de l'expérience, au travers duquel le sujet s'approprie sa maladie. En nous centrant sur l'annonce du diagnostic MCI - soit l'éventualité d'une évolution ultérieure vers la MA - nous avons voulu voir si la parole médicale introduisait effectivement une réorganisation du regard sur la santé et sur les 
perspectives de vie future. Selon notre hypothèse, si les discours des experts de la mémoire comme le diagnostic ne faisaient pas écho aux représentations subjectives des sujets diagnostiqués, les récits de ces derniers n'attribueraient au MCI qu'une place d'élément causal parmi d'autres, voire sans importance, par rapport à leurs perceptions de l'état de santé du patient et de ses perspectives relationnelles. Les postulats épistémiques suivants soutiennent cette approche:

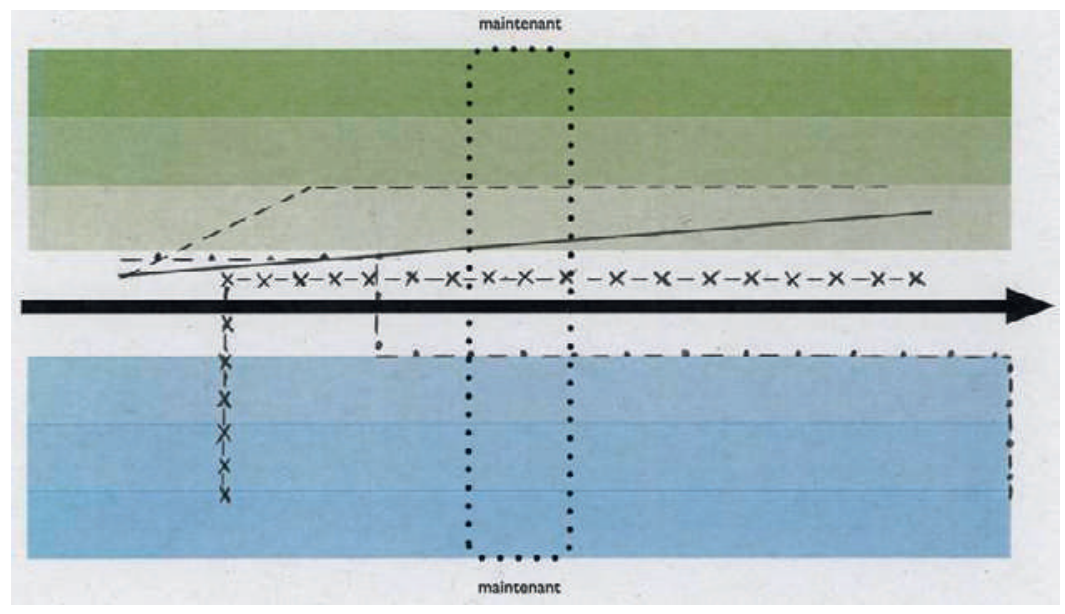

Figure 1: Schéma de représentation graphique de la santé du patient, selon les courbes dessinées par deux patients et leur proche à T1. «Maintenant» indique l'espace correspondant au moment de l'entretien qualitatif. L'axe $\mathrm{x}$ indique le temps. Par souci de se montrer le moins inductif possible, les couleurs de fond ont été choisies dans des tons neutres et nous avons évité de faire figurer sur le schéma les indicateurs «positif» (au-dessus de l'axe temps) et «négatif» (en-dessous de l'axe temps).

Patient 1 / Santé / T1; x_x_Patient 2/ Santé / T1;___._Proche Patient 1 / Santé / T1; ------ Proche Patient 2 / Santé / T1

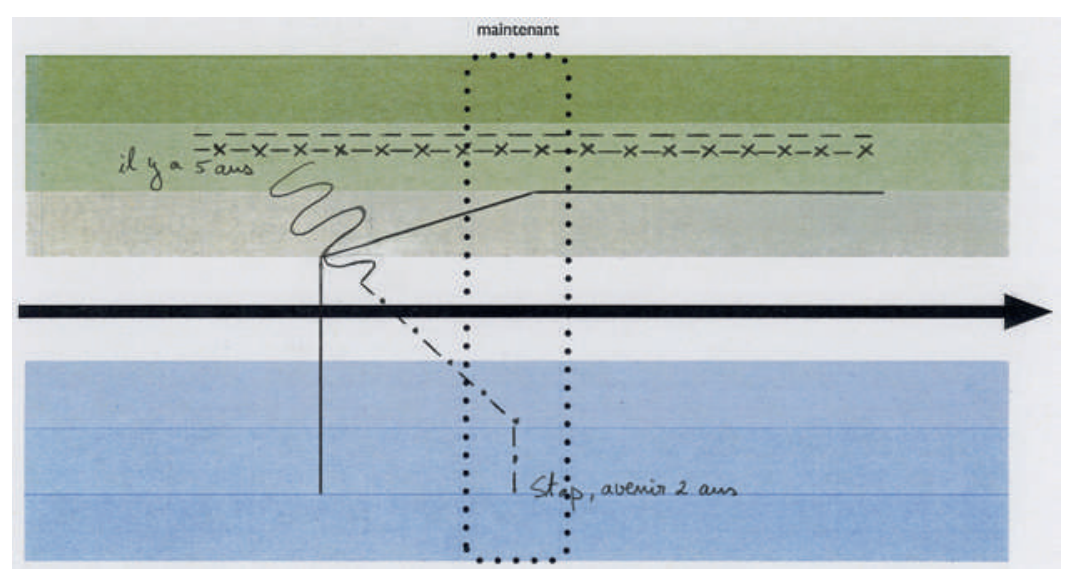

Figure 2: Schéma de représentation graphique de la santé du patient, selon les courbes dessinées par deux patients à T1 et T2. «Maintenant» indique l'espace correspondant au moment de l'entretien qualitatif. L'axe y indique le temps. Les dégradés de couleurs sont volontairement neutres. Les paroles et le zigzag insérés dans le graphique sont rajoutés par le patient 3 luimême.

_ Patient 3 / Santé / T1; x_x_ Patient 4/ Santé / T1;_._._Patient 3 / Santé / T2;

----- Patient 4 / Santé / T2
- Chaque récit est analysable en termes de séquences successives de trois formes de base de récit: progrès, déclin ou état stable.

- Chaque récit possède sa propre temporalité et s'ordonne autour de ses propres moments de tournant (unique ou multiples), lesquels peuvent se situer dans un temps plus ou moins lointain. Le regard porté par le narrateur sur cette trajectoire détermine la courbe qu'il donnera lui-même à sa représentation de son schéma de santé et à sa situation relationnelle.

- Par-delà leur fonction descriptive, les récits et les schémas qui les complètent comportent une fonction identitaire, relationnelle et heuristique [6, 7]. Ainsi, une superposition des courbes rassemblées auprès des différents répondants - dans notre cas les dyades patient-e et proche - devrait permettre de retracer une chronologie subjective du glissement dans la maladie, ainsi qu'indiquer l'effet sur la vie quotidienne de l'assimilation d'un tel diagnostic.

\section{Méthode}

Huit dyades de patients et leurs proches sont rencontrées séparément - soit 8 patient-e-s, 5 femmes et 3 hommes entre 64 et 86 ans, 3 filles de patientes, une belle-fille, 2 époux, une épouse et un partenaire du même sexe - lors de deux séries d'entretiens: une première fois (T1) au plus proche du moment de l'annonce du diagnostic, la seconde (T2) après 4 mois, intervalle de temps devant permettre la mise en évidence de l'évolution ou de la stabilité des récits suite au passage à la consultation mémoire. Le canevas d'entretien est centré sur les motivations des répondants pour solliciter la consultation de la mémoire, sur ce qui y a été fait et sur l'opinion des répondants à propos de ce qui leur a été communiqué à cette occasion. Des signes d'appropriation du risque d'une évolution vers la MA ou d'un autre type de démence, et se manifestant par des récits relatant des réaménagements pratiques dans la vie quotidienne, devaient être un indicateur de la pertinence de l'hypothèse de l'influence de la parole experte sur la narration des patients et des proches. En complément aux entretiens semi-directifs d'environ une heure en tête-à-tête, un support graphique simple, élaboré pour les besoins de cette étude, est présenté à chacun des répondants en leur offrant de dresser leur propre représentation graphique d'une part de leur appréciation respective de la santé du patient et, d'autre part, de leur cercle relationnel (voir figures 1 et $2^{3}$ ). 


\section{Résultats}

Le corpus de données recueillies dans le cadre de ce projet consiste en 30 entretiens discursifs qualitatifs et 58 schémas graphiques ( 2 par répondant et par entretien. Une dyade a refusé de dessiner un schéma).

Le constat le plus marquant est l'absence d'attention prêtée au $\mathrm{MCI}$ en tant qu'entité diagnostique relative à un état de troubles cognitifs. Mis à part le récit de deux filles de patients, le MCI n'est pas le descripteur significatif retenu pour désigner l'état cognitif du ou de la patient-e, ni n'est évoqué en rapport avec les projections sur l'évolution possible de l'état de santé. Si les soucis de mémoire sont avancés comme motif premier de visite à la consultation mémoire, les troubles de mémoire ne sont mentionnés par la suite que sous guidage des enquêteurs. Parlant de leur santé, les patients se montrent plus préoccupés par les affections somatiques dont ils souffrent et ont tendance à minimiser leurs troubles de mémoire. En d'autres termes, le résultat principal de cette étude infirme l'hypothèse de départ puisqu'il montre que la communication du diagnostic MCI n'insuffle pas un tournant dans la trajectoire des patients atteints de troubles cognitifs légers. Les propos des experts lors de l'entretien de restitution semblent n'avoir qu'un faible impact sur la compréhension qu'ont les patients et leurs accompagnants de la santé mentale des sujets diagnostiqués.

Les schémas graphiques n'indiquent pas une réorientation nette des perceptions de santé suite à l'annonce du diagnostic. Ils montrent des patients unanimement optimistes quant à leur santé à T1, soit envisageant une amélioration, soit un état stable. A T2, on trouve chez trois répondants une santé évaluée de manière plus négative par le passé qu'actuellement, trois se situent dans un état de stabilité - un en-dessous de la ligne horizontale qui départage pessimisme d'optimisme, les deux autres au-dessus. En tout, cinq répondants sur les sept se situent dans la zone positive du schéma. Seul un d'entre eux décrit une dégradation par rapport au

Des spécialistes en sciences humaines et sociales réalisent, dans des hautes écoles et des instituts cliniques suisses, des projets de recherche, dont les résultats sont importants pour I'activité médicale. Au printemps 2014, les Académies suisses des sciences ont lancé un appel d'offres dans le but de démontrer le bénéfice de ces recherches pour la pratique médicale et de soins et de faire connaître les principaux résultats des recherches dans le domaine des "Medical Humanities". Les quatre premiers travaux seront publiés dans le BMS. Informations sur les lauréats sous www. akademien-schweiz.ch/medhum passé et marque d'un «stop» ses projections pour les deux ans à venir.

Du côté des proches, l'inquiétude est à peine plus affirmée. A T1, ils sont quatre sur sept à envisager une amélioration (3) ou une stabilité (1) de l'état de santé de leur parent. A T2, seul un des 7 dessine une amélioration de l'état de santé, deux une stabilité, un l'envisageant consécutivement à un déclin. Deux proches évoquent explicitement une dégradation. Enfin, deux autres pronostiquent différents scénarii, tous situés sous la barre horizontale pour l'un, au-dessus de celle-ci pour l'autre. A noter que 13/15 graphiques patients et $8 / 14$ graphiques proches considèrent la santé du patient meilleure ou identique au moment des entretiens de recherche que par le passé.

Deux filles de patientes commentent le diagnostic avec un degré de précision médicale, montrant ainsi une intégration de cette catégorie experte dans leurs représentations de la santé. Les récits et schémas des autres répondants ne font pas référence explicite au $\mathrm{MCI}$ et, contrairement à notre hypothèse de départ, la dimension temps n'opère pas une accentuation de la perception de déclin que l'annonce d'un tel diagnostic aurait pu laisser présager.

Dans l'ensemble, les personnes qui se sont rendues à une consultation mémoire ne semblent pas avoir compris l'évaluation experte établie et elles ne lui accordent pas ou peu de place dans leurs schémas de représentations. Le risque d'une potentielle évolution vers la démence n'est pas entendu. A l'inverse, sont exprimés les arguments autorisant la minimisation du mal ("ce n'est pas Alzheimer»). La plupart des patients pensent que le processus diagnostique n'est pas achevé et n'ont pas le sentiment d'avoir reçu une information de type diagnostique. Aucun des répondants ne mentionne la séance de réévaluation qui devrait se tenir 6 à 12 mois plus tard. On ne constate pas l'adoption d'une posture de malade de la part des patients qui ont consulté.

\section{Relations des résultats de la recherche à la pratique médicale en consultation mémoire}

Les consultations mémoire, implantées en Suisse depuis les années 1990, ont pour mission d'évaluer les fonctions cognitives de patients pour lesquels il existe une suspicion de syndrome démentiel débutant ou de troubles cognitifs légers. La littérature sur ce domaine situé à l'intersection de la neurologie et de la psychiatrie de l'âge avancé [8], montre une relative homogénéité des cadres de travail propres à ces structures: équipes pluridisciplinaires (médecins 
neurologues et/ou gériatres et/ou psychiatres, neuropsychologues, infirmières et/ou travailleuses sociales), similarité organisationnelle (démarche en 4 étapes: anamnèse, hétéro-anamnèse, tests neuropsychologiques de dépistage et examens complémentaires, notamment IRM). Ce travail d'harmonisation résulte des conférences de consensus successives qui ont dégagé un consensus sur les critères de diagnostic basés sur la classification CIM-10 [9] ou du DSMIV-TR [10] et sur les procédures permettant de retenir la présence possible ou probable de tels syndromes. Ces efforts sont soutenus par le souci d'actualiser les connaissances sur ces phénomènes complexes. Depuis son adoption dans les années 1980, le diagnostic MCI ne cesse en effet de susciter des débats experts autour des difficultés de distinction entre signes d'un syndrome démentiel débutant, dont celui d'une MA, d'un vieillissement normal. Conséquence des inconnues qui persistent quant à l'étiopathologie des troubles démentiels liés à l'âge, la fonction indicative du MCI reste sujette à controverses: est-il la désignation d'un stade précoce de la MA, de troubles de type Alzheimer, ou est-ce un diagnostic syndromique empreint d'incertitudes? Pour certains [1-13], ce diagnostic n'a d'utilité qu'à condition d'être réservé à un stade pré-démentiel de MA, estimant que sa trop grande hétérogénéité limite son utilité pour un diagnostic précoce. Outre le débat sur le spectre d'application du MCI, les cliniques de la mémoire se distinguent entre elles dans les moyens techniques dont elles disposent (recours à l'IRM, PET scan, analyse du liquide céphalorachidien etc.), différences dans les moyens d'analyse médicale ayant bien souvent à la base des différences de ressources financières. Et enfin, les contextes socioculturels jouent également un rôle, avec des sensibilités différentes aux troubles cognitifs des personnes âgées.

Nous estimons que l'indifférence de nos répondants patients et, dans une large mesure, proches - au MCI à la suite de l'annonce du diagnostic est à mettre en relation avec l'état de la question. Si le phénomène n'avait été observé que chez les patients, on aurait pu supposer qu'il s'agit d'amnésie ou d'anosognosie. Etant donné que les proches réagissent à l'identique, il est légitime de mettre le phénomène en relation avec les controverses entourant le $\mathrm{MCI}$ ainsi qu'avec la façon de transmettre le diagnostic. On peut en effet supposer que le positionnement du clinicien face au débat qui entoure le statut du MCI n'est pas étranger à sa spécialisation - psychiatrie ou neurologie par exemple - et que ceci influence sa manière de le poser et de l'annoncer. Les colloques de synthèses au cours desquels les équipes déterminent les réponses à donner aux patients ${ }^{4}$ nous ont fait voir de grands écarts dans l'attribution du MCI, allant de l'intention de communiquer un diagnostic "intermédiaire» ayant la fonction de préparer à la suite (démence), au refus de mentionner explicitement le MCI jugeant qu'il ne s'agit pas d'une information utile pour le patient. Il existe en effet un débat soutenu parmi les cliniciens sur les possibles conséquences de la transmission d'un diagnostic MCI [8]. Les narrations de nos répondants nous laissent supposer que le flou de la catégorie se reflète dans la manière dont le diagnostic leur a été annoncé. Du point de vue des patients et de leurs proches interviewés, le MCI a tout l'air d'un non-diagnostic en dépit de la procédure structurée de recueil des données.

Un second constat soutenant nos observations d'indifférence au diagnostic tient au grand fractionnement de la démarche diagnostique: le patient et le proche sont interrogés séparément par deux types d'intervenants différents, le médecin qui a mené l'entretien d'anamnèse n'est pas présent lors de la phase conséquente des tests neuropsychologiques, mais c'est à lui qu'il appartient de faire un retour au patient. Quant au médecin traitant, qui est souvent à l'origine de l'orientation du patient vers la consultation mémoire, il est totalement absent du processus. Les patients ainsi que les proches rendent compte de cette dispersion des intervenants cumulée à l'étalement de la procédure sur un long laps de temps, par leur incapacité à retracer les grandes étapes de leur parcours au sein de la consultation mémoire. Ils ne parviennent pas non plus à préciser les fonctions professionnelles des cliniciens rencontrés au cours du parcours.

Une troisième difficulté pour les patients et proches à saisir le sens du MCI tient au fait que celui-ci fonctionne comme diagnostic par élimination: on l'attribue lorsqu'on n'est pas en mesure de formuler un état par l'affirmative («Vous avez une démence débutante»). Ainsi, les patients de notre échantillon se positionnent entre l'indifférence («On ne m’a rien dit») et la satisfaction de n'avoir rien entendu de grave («Non, ce n'est pas Alzheimer», ainsi que la fille d'un des patients, consciente qu'il s'agit de l'annonce d'une possible évolution vers la démence, trouve préférable de communiquer à son père). La récurrence de ces expressions d'apaisement vis-à-vis de la crainte de la MA qui demeure dans l'arrière-fond implicite des récits de nos répondants laisse supposer que la manière dont le $\mathrm{MCI}$ est restitué rend cette interprétation possible. 


\section{Recommandations généralisables}

Patients et proches n'ont pas perçu l'utilité de la démarche suivie à la consultation mémoire. Il serait utile de vérifier si la portée préventive du MCI - soit l'introduction précoce de stratégies visant l'amélioration de la qualité de vie des patients et de leur entourage [10] - se verrait augmentée si le message communiqué posait d'emblée le statut transitoire et incertain de ce diagnostic, non pas comme une faiblesse d'évaluation de celui-ci, mais comme la particularité de ce statut. Afin de vérifier cette hypothèse, nous prévoyons une recherche consécutive à celle-ci qui se concentrera sur les séances de communication du diagnostic aux patients et à leurs proches.

Le diagnostic des troubles de la mémoire confère aux proches un espace significatif dans le processus. Il est apparu que les proches ne sont pas au clair sur la place accordée à leurs propos dans la détermination du diagnostic. Une approche systémique dans une perspective psychoéducative au domicile du patient pourrait contribuer à préciser à tous les acteurs engagés les enjeux des interventions des uns et des autres. Des expériences cliniques dans ce sens seraient utiles à la vérification de cette question.

Et enfin une réflexion de fond: les difficultés rencontrées dans le cadre de cette étude pour réunir un échantillon d'à peine dix patients nous amène à penser que c'est la liminalité de la catégorie $\mathrm{MCI}$ qui empêche les cliniciens d'en faire usage. Il nous apparaît que la solution au problème n'est pas de l'ordre d'un choix entre deux approches: soit réserver le MCI pour les phases prodromales de la MA tel que cela est réclamé par la tendance de Dubois, soit tenir compte des aspects sociaux et subjectifs dans les critères d'application de la catégorie. La population concernée le plus souvent par le diagnostic MCI est constituée de personnes affectées par une multitude de syndromes susceptibles chacun à leur façon d'altérer les fonctions cognitives. C'est pourquoi nous suggérons que l'évaluation des troubles cognitifs se distancie d'une logique basée sur une vision unitaire du phénomène.
Notes de fin

1 Le trouble cognitif léger est une catégorie diagnostique à risque élevé d'évolution vers une démence de type Alzheimer ( $\mathrm{MCI}$ amnésique) ou un autre type de démences (MCI non amnésique). Le diagnostic est établi sur la base d'éléments anamnestiques fournis par le patient et ses proches, et sur l'évaluation clinique et des fonctions cognitives [1].

2 FNS/DORE 13DPD6 134989, Fondation Leenaards N/réf 2977.5/ss.

3 Par souci de place, nous reproduisons ci-dessous un choix de gra phiques portant sur les perceptions de la santé d'uniquement quatre patients différents et celles des proches de deux d'entre eux.

4 Des observations répétées de ces colloques ont été faites au cours de la recherche.

\section{Références}

1 Leuba G, Büla C, Schenk F. (ed). Du vieillissement cérébral à la maladie d'Alzheimer. Vulnérabilité et plasticité. Bruxelles: De Boeck, 2003, ch. 8

2 Corcuff P. Les nouvelles sociologies. Paris: Armand Colin; 2005

3 Whitehouse P, George D. The myth of Alzheimer's: what you aren't being told about today's most dreaded diagnosis. New York: St. Martin's Press, 2008.

4 Maisondieu J. Le crépuscule de la raison: Comprendre pour les soigner les personnes âgées dépendantes. Paris: Bayard Editions; 1989

5 Gergen KJ. Construire la réalité: un nouvel avenir pour la psychothérapie. Paris: Seuil, 2005.

6 Bruner J. Actual minds, possible worlds. Cambridge Mass. London: Harvard University Press; 1986.

7 Bruner J. Pourquoi nous racontons-nous des histoires?. Paris: Pocket, 2005.

8 Lin JS, O'Connor E, Rossom R, et al. Screening for Cognitive Impairment in Older Adults: An Evidence Update for the U.S. Preventive Services Task Force. Evidence Report: Agency for Health Care Research and Quality; 2013.

9 Braekhus A, Ulstein I, Wyller TB, et al. The Memory clinic outpatient assessment when dementia is suspected. Tidsskr Nor Laegeforen. 2011;22(131):2254-7.

10 Gremaud Brulhart F, Decrey H, Démonet J-F, et al. Troubles de la mémoire chez la personne âgée: que faire au cabinet? Revue médicale suisse. 2013;9:2029-33.

11 Michel BF, Becker H. Mild Cognitive Impairment, Stade précoce de la maladie d'Alzheimer? Neurologie. 2002;5:125-29.

12 Dubois B, Albert ML. Amnestic MCI or prodromal Alzheimer's disease? Lancet Neurol. 2004;3(4):246-8.

13 Acket B, Lemesle B, Puel M, et al. Que reste-t-il du MCI en 2009? Neurologie.com. 2009;1(1)

- Association américaine de psychiatrie. DSM-IV: diagnostic and statistical manual of mental disorders. Washington DC: American Psychiatric Association, 1995, 4th ed.

- Organisation mondiale de la santé. Classification internationale des maladies. Dixième révision. Chapitre $\mathrm{V}(\mathrm{F})$ : troubles mentaux et troubles du comportement: critères diagnostiques pour la recherche. Genève: World Health Organisation, 1993. 